\title{
Mapping Polarization of Perovskite Oxides across Scales Using 4D STEM with Improved Spatial Resolution
}

\author{
Tim Eldred ${ }^{1}$, Jacob Smith ${ }^{1}$, Stephen Funni ${ }^{2}$, Elizabeth Dickey ${ }^{2}$ and Wenpei Gao ${ }^{1}$ \\ ${ }^{1}$ North Carolina State University, United States, ${ }^{2}$ Carnegie Mellon University, United States
}

The application of four-dimensional scanning transmission electron microscopy (4DSTEM) in Nanobeam Electron Diffraction (NBED) has enabled studies to correlate local nanoscale structure with microscale phenomena. The study of the phase and domain structures of Barium Titanate $\left(\mathrm{BaTiO}_{3}\right.$, BTO) is an example of how the material properties can be engineered with improved understanding on the complexity in structure as the spatial resolution increase. Prior works $[1,2]$ have demonstrated that nominally tetragonal regions are more complex, containing offset rhombohedral nanoregions and clusters even at temperature ranges that predict tetragonal structure. The advent of 4DSTEM has allowed mapping of projected polarization and phase at spatial resolutions limited only by the probe size. Since the studies on mapping BTO phases rely on the analysis of the non-overlapping structure of the disks in convergent beam electron diffraction (CBED), the sampling resolution is limited by the probe sizes $(0.8 \sim 1.5 \mathrm{~nm})$ corresponding to the convergence angles below the Bragg angle, resulting in probes sampling multiple columns of unit cells. To improve spatial resolution requires using higher convergence angles, however, complications of the intensity in CBED with overlapping regions challenges data analysis.

In this presentation, we report the mapping of the polarization, orientation and phase in BTO sampled at a rate equivalent to the size of unit cells in two dimension (2D) using 4DSTEM. We accomplish this by taking advantage of the fact that the intensity structure populated within the non-overlapping region of a partially overlapped CBED is equivalent to that from the CBED patterns acquired using a small convergence angle equal to that area. By increasing the convergence angle to above the Bragg angle, we generate slightly overlapping CBED patterns, resulting in probe sizes at or below the dimension of the unit cell, allowing the sampling of a single column of unit cells, analyzing the projected symmetry. Masking the overlapping regions in CBED separates the diffraction disks and allows for analysis of the non-overlapping fractions equivalent to those acquired using lower convergence angles, as demonstrated in Figure 1. Intensity analysis of Friedel pair diffraction spots or symmetry analysis of the diffraction pattern can be performed. We will demonstrate the equivalency of intensity distributions within the lower angle of the CBED disks with simulations performed on both polar and non-polar BTO structures, as well as with experimental results.

Experimental 4DSTEM data was obtained with an EMPAD on a Thermo Scientific Titan aberration-corrected microscope operated at $200 \mathrm{kV}$. A single crystal BTO sample was mechanically polished and thinned using argon ion milling (Gatan PIPSII). The data acquisition was performed at semi-convergence angles ranging between 2 and $4 \mathrm{mrad}$ with a sampling rate of a $0.24 \mathrm{~nm}$ step size for 4DSTEM. Atomic resolution high angle annular dark field (HAADF) images were also taken using a semi-convergence angle of 17.9 mrad. Multislice and Bloch wave methods were used to simulate 4DSTEM and CBED patterns of BTO for comparison with experimental data.

With the improved resolution and flexibility in data analysis, the results show an evolution of the structural information across spatial scales, presented in Figure 2. The unit cell level data shows local, regions of shifting projected polarization, in contrast with the more uniform transition in projected polarization observed with larger probe sizes. This data will be compared with a mapping using the center of mass (COM) in the center disk of the diffraction. [3] 

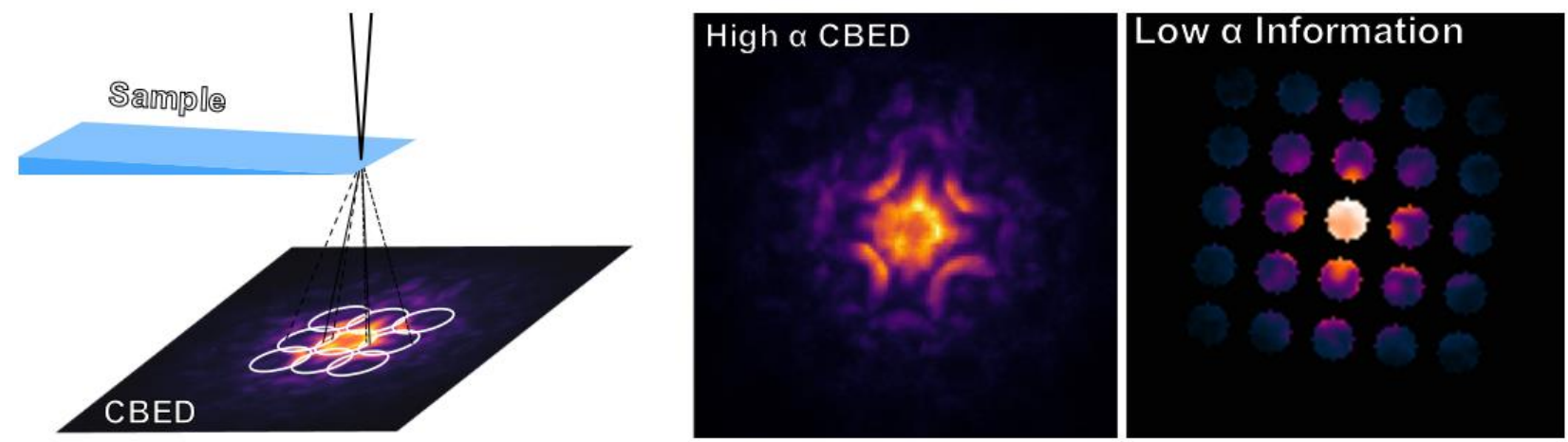

Figure 1. NBED 4DSTEM Data acquisition scheme (Left), with example partially converging CBED patterns (Middle) and example masked data (Right) showing the retained information within the low-semi convergence angle.
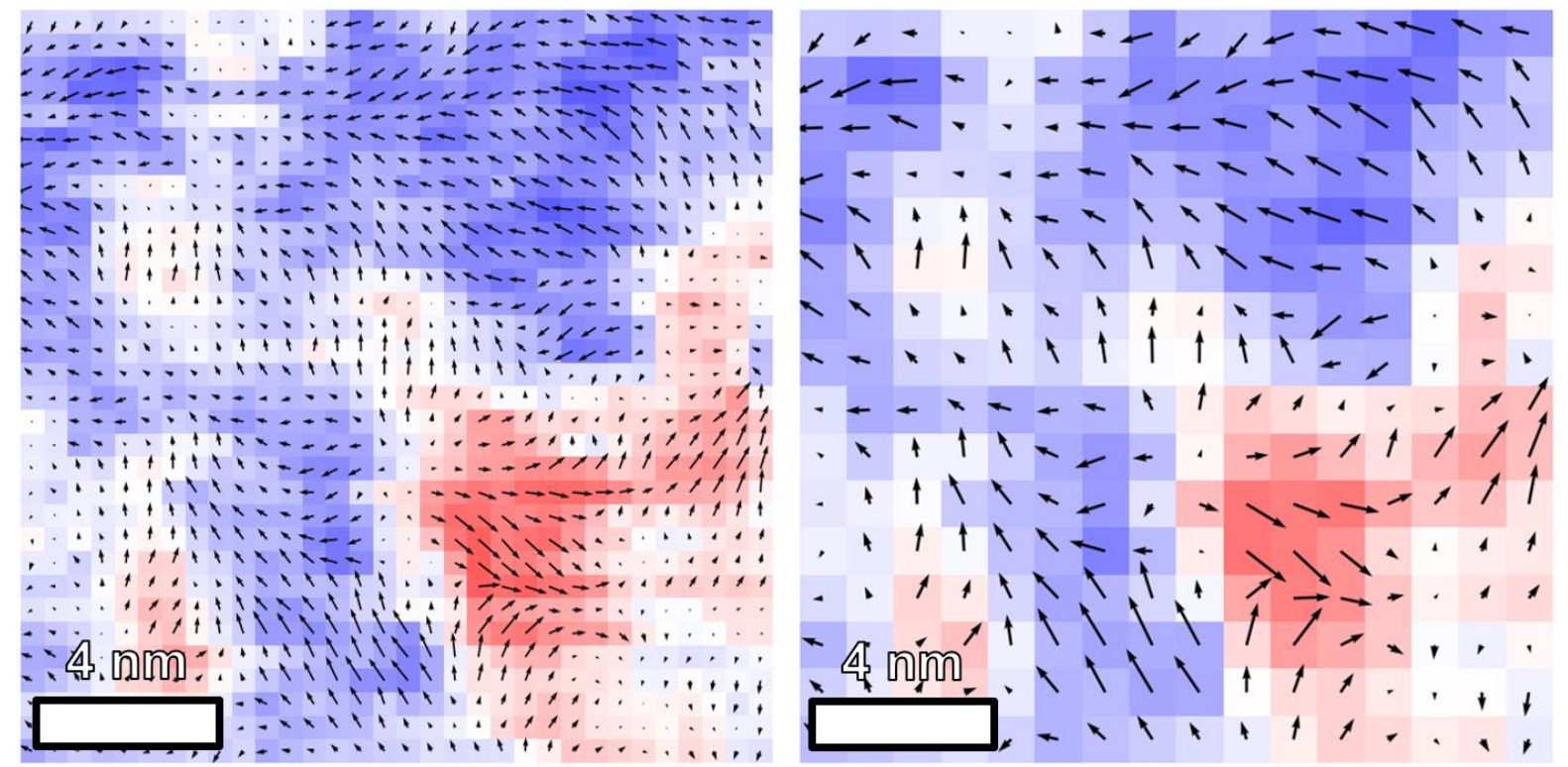

Figure 2. Vector map calculated using $(\mathrm{I}(020)-\mathrm{I}(0-20)) / \overline{\mathrm{I}}\{020\}$ and $(\mathrm{I}(002)-\mathrm{I}(00-2)) / \overline{\mathrm{I}}\{002\}$ from the diffraction pattern at the unit cell level (left) and over $2 \times 2$ unit cells (right).

\section{References}

[1] Tsuda, K.; Yasuhara, A.; Tanaka, M. Two-Dimensional Mapping of Polarizations of Rhombohedral Nanostructures in the Tetragonal Phase of BaTiO3 by the Combined Use of the Scanning Transmission Electron Microscopy and Convergent-Beam Electron Diffraction Methods. Appl. Phys. Lett. 2013, 103 (8), 082908. https://doi.org/10.1063/1.4819221.

[2] Shao, Y.-T.; Zuo, J.-M. Nanoscale Symmetry Fluctuations in Ferroelectric Barium Titanate, BaTiO3. Acta Cryst B 2017, 73 (4), 708-714. https://doi.org/10.1107/S2052520617008496.

[3] This work was supported by start-up fund from the College of Engineering and Department of Materials Science and Engineering at North Carolina State University. Electron microscopy was performed at the Analytical Instrumentation Facility (AIF) at North Carolina State University, which is supported by the State of North Carolina and the National Science Foundation (award number ECCS-2025064). The AIF is a member of the North Carolina Research Triangle Nanotechnology Network (RTNN), a site in the National Nanotechnology Coordinated Infrastructure (NNCI). 